\title{
Expanding the phenotype of GNAO1-related movement disorders: two cases with mild phenotypes.
}

\author{
Amal Abu Libdeh, $\mathrm{MD}^{1 \oplus}$ and Albert Chang ${ }^{2}$ \\ ${ }^{1}$ Department of Neurology, University of Virginia, Charlottesville, USA \\ ${ }^{2}$ School of Medicine, University of Virginia, Charlottesville, USA \\ Corresponding author: Amal Abu Libdeh; aa9qu@ virginia.edu \\ do) https://doi.org/10.17724/jicna.2020.168
}

Received: 9 September 2020

Accepted: 9 October 2020

\begin{abstract}
Background: Pathogenic variants in the GNAOl gene have been linked to severe epileptic encephalopathy, severe progressive movement disorders, developmental delay, and intellectual disability. The majority of cases of GNAOI variants reported in the literature exhibit severely debilitating phenotypes.

Method: Reporting on two cases.

Results: We present two unrelated girls, an 18-year-old, and a four and a half-year-old, with mild phenotypes. While each was noted early on to have hypotonia, failure to thrive, and developmental delay, they have both made significant progress with therapeutic interventions. Neither have had any developmental regression, seizures or choreoathetosis.

Conclusion: These two cases further expand the phenotype spectrum of GNAO1 mutations, which is important for counseling and to help guide management plans.
\end{abstract}

Keywords: GNAOI, G protein, exome sequencing, epileptic encephalopathy, movement disorders, abnormal gait.

(C) Libdeh A.A. \& Chang A.; licensee JICNA

\section{Introduction}

Guanine-binding protein (G-protein)-coupled receptors modulate neuronal excitability. Heterotrimeric G-proteins are composed of a-, b-, and g-subunits. The most abundant class of Ga-subunits is called Gao and is encoded by the GNAOl gene [1]. Variants in this gene have previously been linked to severe epileptic encephalopathy and severe hyperkinetic disorders. Patients are typically hypotonic and have significant intellectual disability [2]. The majority of cases of GNAOl variants reported in the literature exhibit severely debilitating phenotypes. Further characterisation of milder clinical phenotypes is crucial in elucidating the full spectrum of GNAO1-related disorders.

\section{Case reports}

Case 1

An 18-year-old girl presented to the clinic with dysphonia and abnormal gait. Following an uneventful perinatal history, she was noted to have had low tone early on in life. At nine months of age, she was falling behind in developmental milestones with failure to thrive. As a result, therapies (physical, occupational and speech) were started, and she made steady progress over time. At 20 months of age, she began to walk. She is now fully ambulatory without limitation. She is able to speak fluently, but also has dysarthria that can occasionally make her speech difficult to understand. Talking slowly and taking deep breaths before she speaks tend to help make her speech more understandable. Occasionally, she has mild drooling, but no choking or difficulty swallowing. Her speech and her gait worsen when fatigued. She actively participates in sports, including frisbee, soccer and hiking. She volunteers as a camp counselor and is able to drive. She had an Individualised Education Plan (IEP) early on in her schooling but did not need one in high school or thus far in college. Her condition plateaued in severity over the last few years. She has not had any developmental regression, seizures or dyskinetic movements. She has been diagnosed with chronic migraine without aura. She takes amitriptyline and riboflavin for prophylaxis, and zolmitriptan as needed for rescue. Regarding family history, the patient has one brother who has migraines and is otherwise healthy. There is no further family history of developmental or neurological disorders. When she was examined in clinic, she had no abnormalities on her general exam. Her weight was in the $60^{\text {th }}$ centile, and her height in the $95^{\text {th }}$ centile. Her neurological exam showed normal cranial nerve function, except for dysphonia, and mild tongue tremor. Appendicular tone was mildly decreased throughout, and strength was full. Deep tendon reflexes were +2 and symmetrical throughout, and flexor plantar response was intact. Her gait was narrow-based, with a mildly 
stooped posture, decreased knee-bending, and genu valgum. Additionally, arm swing was noted to be decreased bilaterally with mild dystonic flexion of both arms. (See video.)

Throughout her life she has had extensive work-up which was unrevealing. Tests included normal creatine kinase, normal metabolic work-up (serum quantitative amino acids, urine organic acids), normal thyroid function test, negative antinuclear antibodies (ANA), normal immunoglobulin levels, normal coeliac panel, and normal alpha fetoprotein level. A muscle biopsy, electromyography/nerve conduction study (EMG/NCS), and cardiac echocardiogram were normal. A video stroboscopy showed normal vocal cord function. She had two brain magnetic resonance imagings (MRIs), one at the age of two years, and the other at the age of 16 years, both normal. Genetic tests yielded a normal karyotype, a chromosomal microarray was without clinically significant abnormalities, cystic fibrosis-testing was negative, and both facioscapulohumeral muscular dystrophy and myotonic dystrophy-testing was negative. The patient's mother works as a nurse, and she remained interested in finding a diagnosis, so she saw a different neurologist. In addition to ordering the second brain MRI mentioned above, an exome sequencing test was ordered.

The exome sequencing via GeneDx showed the patient to be heterozygous for the de novo $\mathrm{N} 242 \mathrm{~T}$ pathogenic variant in the GNAO1 gene (c.725 A>C, p.Asn242Thr (AAC $>$ ACC)). For this gene, $100 \%$ of the coding region was covered at a minimum of 10x by the XomeDx test. The patient's mother and father were both confirmed to not harbour this variant. The N242T variant in the GNAOI gene has not been reported previously as a pathogenic variant, nor a benign variant. The $\mathrm{N} 242 \mathrm{~T}$ variant is not observed in large population cohorts [3]. The N242T variant is a conservative amino acid substitution, which is not likely to impact secondary protein structure, as these residues share similar properties. Silico analyses, including protein predictors and evolutionary conversation, support a deleterious effect. No other variants were reported in this patient. This particular variant has been reported as a de novo variant with confirmed parentage in an individual previously tested at GeneDx with a GNAOI-related disorder.

\section{Case 2}

A five-year-old girl presented to the clinic with developmental delay and abnormal gait. She had an uneventful perinatal history. At the age of three months, she was noted to have low tone and failure to thrive. She has global developmental delay but has been making steady progress with various therapies (physical, occupational and speech). She first walked at approximately two years of age, but continues to present with intermittent toe-walking, and has frequent falls. She can only say a few words. She has not had any regression in milestones, seizures, or choreoathetosis. The patient has no siblings, and there is no further family history of developmental or neurological disorders. Her general exam was unremarkable. Her weight was in the $70^{\text {th }}$ centile, and her height was in the $95^{\text {th }}$ centile. Her neurological exam showed normal cranial nerve function. Appendicular tone was mildly decreased throughout, but strength was full. Deep tendon reflexes were +2 and symmetrical throughout, and flexor plantar response was intact. Her gait was wide-based, with flexed knees, and she held her arms in flexion as she walked. Work-up included a brain MRI which was normal. Chromosomal microarray was normal, and testing for Rett syndrome and for Prader Willi syndrome were negative.

Most recently, she had exome-sequencing via GeneDx, and that showed the patient to be heterozygous for a de novo pathogenic variant in the GNAO1 gene (C.724-8G>A; IVS6$8 \mathrm{G}>\mathrm{A}$ ). For this gene, $100 \%$ of the coding region was covered at a minimum of 10x. This variant is predicted to damage the natural splice acceptor site, and creates a stronger splice acceptor site in intron 6 . The patient's mother and father were both confirmed to not harbour this variant. No other variants were reported in this patient. This variant has been reported de novo in an individual with abnormality in the nervous system [3]. In addition, GeneDx has previously observed this variant as de novo in two presumably unrelated individuals with developmental delay and dystonia. This variant was not observed in large population cohorts [4].

\section{Discussion}

The GNAOI gene has a cytogenetic location of 16q12.2 and encodes the alpha subunit of the Go heterotrimeric G-protein signal-transducing complex [1]. Variants in this gene are detected using exome-sequencing or targeted next generation sequencing panels. De novo variants in GNAOI were first described in 2013 in four children with severe epileptic encephalopathy and intractable seizures, starting as early as a few days of life [1]. Some of these patients also had dyskinesia. In 2016, the first case reports of patients with severe movement disorders and few or no seizures emerged [2]. Since then, there have been over 60 cases reported in the literature, with more than 20 pathogenic variants identified. These are largely characterised by highly debilitating symptoms, such as epileptic encephalopathy, severe chorea, athetosis and other dyskinesias. Earlier reports suggested a genotype-phenotype correlation where variants leading to loss of function lead to epileptic encephalopathy, and variants leading to gain of function result in a hyperkinetic movement phenotype [5]. That was later challenged, with the recognition that phenotypes fall on a spectrum [6]. In patients with the movement phenotype, exacerbations of the hyperkinetic movements frequently lead to hospitalisation, including admission to intensive care units. Many of these cases are refractory to treatment. Tetrabenazine, topiramate and deep brain stimulation have been used to alleviate symptoms $[7,8,9,10]$. Unlike the two patients described in this report, structural brain abnormalities have been reported, including cerebral atrophy, small caudate, thin or dysmorphic corpus callosum, ventricular enlargement and delayed myelination [11, 12].

The first patient in this report is one of the oldest patients with this variant to be reported in the literature. Previously, a 19-yearold patient was reported with the GNAOI variant (c.625C $>\mathrm{T}$, p.Arg209Cys) $[2,8]$, but she had severe symptoms, including progressive chorea, and was refractory to multiple anticonvul- 
sants. In contrast, our first patient mentioned above successfully made progress with therapy, and currently leads an independent life. Previous reports described cases with a 'mild' phenotype [12], but these patients still had seizures and dyskinesia. Neither of the two patients in this report had seizures or dyskinesia, and they have not required pharmacological treatment. The parents in the first case were interested in reaching a diagnosis, which led to ordering an exome-sequencing, although many patients with such phenotypes do not get tested.

Whole-exome-sequencing (WES) has revolutionised the diagnostic yield in patients with rare conditions, solved many challenging cases, and has helped expand the phenotype of many variants. Given the limited availability of this test, clinicians tend to reserve it for patients with severe symptoms, especially when having a clear diagnosis would change management. However, patients with milder symptoms from a genetic variant are less likely to get tested, ultimately failing to contribute to the phenotypic spectrum of the respective variant type. A clinician's experience, the parents' motivation, the availability of genetic counselors, and insurance coverage are the main factors that influence whether exome-sequencing is performed. As exome-sequencing becomes more readily available, patients will be tested more often, and starting at a relatively younger age. It is of utmost importance to know the spectrum associated with rare genetic variants in order to provide proper counseling and anticipatory guidance to patients.

\section{Disclosures}

The authors declare no funding sources or conflicts of interest to disclose. The authors report no financial disclosures for the previous 12 months.

\section{Author contributions}

Amal Abu Libdeh: Contributions include clinical data collection, data analysis and interpretation, conception of the case reports, critical revision and critique of the article, and final approval of the version to be published.

Albert Chang: Contributions include data analysis and interpretation, writing of the first draft, critical revision and critique of the article, and final approval of the version to be published.

\section{Ethical compliance statement}

We confirm that we have read the journal's position on the issues involved in ethical publication and affirm that this work is consistent with those guidelines. Informed consent was obtained from both families of the two cases described in the report. Written consent allowing for the inclusion of the media file was provided by the respective patient's family. Verbal consent was obtained from both families for the use of patient data in both case reports. We confirm that the approval of an institutional review board was not required for this work.

\section{Competing interests}

None.

This is an Open Access article distributed under the terms of the Creative Commons Attribution License (http://creativecommons.org/licenses/by/4.0), which permits unrestricted use, distribution, and reproduction in any medium, provided the original work is properly credited. The Creative Commons Public Domain Dedication waiver (http://creativecommons.org/publicdomain/zero/1.0/) applies to the data made available in this article, unless otherwise stated.

\section{Cite this article as:}

Libdeh A.A. \& Chang A. (2020). Expanding the phenotype of GNAO1-related movement disorders: two cases with mild phenotypes. Journal of the International Child Neurology Association, 20(168). https://doi.org/10.17724/jicna.2020.168

\section{References}

[1] Nakamura K, Kodera H, Akita T, Shiina M, Kato M, Hoshino H, et al. De Novo Mutations in GNAO1, Encoding a Gao Subunit of Heterotrimeric G Proteins, Cause Epileptic Encephalopathy. The American Journal of Human Genetics. 2013;93(3):496-505. PubMed.

[2] Saitsu H, Fukai R, Ben-Zeev B, Sakai Y, Mimaki M, Okamoto N, et al. Phenotypic spectrum of GNAO1 variants: epileptic encephalopathy to involuntary movements with severe developmental delay. European Journal of Human Genetics. 2015;24(1):129-134. PubMed.

[3] Retterer K, Juusola J, Cho MT, Vitazka P, Millan F, Gibellini F, et al. Clinical application of whole-exome sequencing across clinical indications. Genetics in Medicine. 2015;18(7):696-704. PubMed.

[4] Lek M, , Karczewski KJ, Minikel EV, Samocha KE, Banks $\mathrm{E}$, et al. Analysis of protein-coding genetic variation in 60, 706 humans. Nature. 2016;536(7616):285-291. PubMed.

[5] Feng H, Sjögren B, Karaj B, Shaw V, Gezer A, Neubig RR. Movement disorder in GNAO1 encephalopathy associated with gain-of-function mutations. Neurology. 2017;89(8):762-770. PubMed.

[6] Pearson TS, Helbig I. Epileptic encephalopathy, movement disorder, and the yin and yang of GNAO1 function. Neurology. 2017;89(8):754-755. PubMed.

[7] Kulkarni N, Tang S, Bhardwaj R, Bernes S, Grebe TA. Progressive Movement Disorder in Brothers Carrying a GNAO1 Mutation Responsive to Deep Brain Stimulation. Journal of Child Neurology. 2015;31(2):211-214. PubMed. 
[8] Sakamoto S, Monden Y, Fukai R, Miyake N, Saito H, Miyauchi A, et al. A case of severe movement disorder with GNAO1 mutation responsive to topiramate. Brain and Development. 2017;39(5):439-443. PubMed.

[9] Dhamija R, Mink JW, Shah BB, Goodkin HP. GNAO1 -Associated Movement Disorder. Movement Disorders Clinical Practice. 2016;3(6):615-617. PubMed.

[10] Koy A, Cirak S, Gonzalez V, Becker K, Roujeau T, Milesi $\mathrm{C}$, et al. Deep brain stimulation is effective in pediatric patients with GNAO1 associated severe hyperkinesia. Journal of the Neurological Sciences. 2018;391:31-39. PubMed.

[11] Dietel T, Evers C, Hinderhofer K, Korinthenberg R, Ezzo D, Bönnemann C, et al. Expanding Phenotype of De Novo Mutations in GNAO1: Four New Cases and Review of Literature. Neuropediatrics. 2017;48(05):371-377. PubMed.

[12] Danti FR, Galosi S, Romani M, Montomoli M, Carss KJ, Raymond FL, et al. GNAO1 encephalopathy. Neurology Genetics. 2017;3(2):e143. PubMed.

\section{Video legend}

Video: Case 1 exam findings, including abnormal speech, and abnormal gait. 